\title{
Interview with Amitai Regev
}

Toufik Mansour

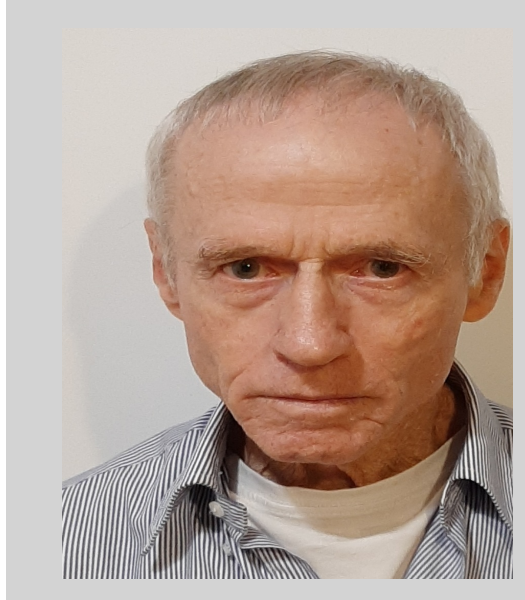

\begin{abstract}
Amitai Regev is the Herman P. Taubman Professor of mathematics at the Weizmann Institute of Science. He received his doctorate from the Hebrew University of Jerusalem in 1972, under the direction of Shimshon Amitsur. Regev has made significant contributions to the theory of polynomial identity rings. He developed the so-called "Regev theory" that connects polynomial identity rings to representations of the symmetric group, and hence to Young tableaux. He has made seminal contributions to the asymptotic enumeration of Young tableaux and tableaux of thick hook shape, and together with William Beckner proved the Macdonald-Selberg conjecture for the infinite Lie algebras of type B, C, and D.
\end{abstract}

Mansour: Professor Regev, first of all, we would like to thank you for accepting this interview. What do you think about the development of the relations between combinatorics and the rest of mathematics?

Regev: Often there is an algebraic structure with a corresponding combinatorial structure, which is induced by the corresponding algebra structure.

Mansour: What have been some of the main goals of your research?

Regev: The goal of my research has been to find and to better understand the polynomial identities of algebras, in particular, what are the polynomial identities of matrices over a field. For instance, the tensor product of two PI algebras is still a PI algebra.

Mansour: What were your early experiences with mathematics?

Regev: I essentially had no math before university. At school (in a Kibutz) I did notice that I can do most math problems easily, hence for university I decided to try math.

Mansour: Were there specific problems that made you first interested in combinatorics?
Regev: For my Ph.D. ${ }^{1}$ research (under Amitsur) I chose the $A \otimes B$ problem, which was then open. At an early stage of that research, I realized I should determine the case $G \otimes G$ where $G$ is the Grassmann algebra ${ }^{2}$. After that, plus lots of work, the general case - followed. I arrive in combinatorics later in my mathematical career, so I feel lack the overview.

Mansour: What was the reason you chose the Hebrew University for your Ph.D. and your advisor, Shimshon Amitsur?

Regev: At that time I thought I was mostly interested in algebra. Algebra in Israel at that time meant algebra at the Hebrew University, and mostly - with Amitsur.

Mansour: How was the mathematics in Israel and specifically at the Hebrew University at that time?

Regev: In ring theory, the math department at the Hebrew University was one of the strongest in the world, and much better than other departments in Israel. Here I would mention Avinoam Mann, who substituted Amitsur as my Ph.D. advisor during the times Amitsur was abroad.

The authors: Released under the CC BY-ND license (International 4.0), Published: January 1, 2021

Toufik Mansour is a professor of mathematics at the University of Haifa, Israel. His email address is tmansour@univ.haifa.ac.il

${ }^{1}$ See, A. Berele and Y. Roichman, The mathematics of Amitai Regev, Advances in Applied Mathematics 37 (2006) $132-138$.

${ }^{2}$ See, A.P. Popov, Identities of the tensor square of the Grassmann algebra, Algebra Logic 21 (1982) 296-316 
Mansour: Would you say a little bit more about your most influential results and why they have been influential?

Regev: Codimensions, cocharacters and growth were introduced in my thesis (see footnote ${ }^{1}$ ). To see their role in ring theory let me quote from Formanek's review of a paper of mine on growth: "The theory of polynomial identities ring (PI rings) can be divided into two parts, a ring theoretic part, which studies properties of PI rings, and a quantitative part which studies the $T$-ideal of identities. Until about 1985, the greater part of research in PI rings was of the former type, but since then the second is predominated." Growth was introduced and studied in my thesis and was extended by several authors.

The Schur-Weyl duality is of fundamental importance in all areas of math, in particular in combinatorics. The asymptotics of corresponding combinatorial sums is essential in that study. With my student Allan Berele we developed a $Z_{2}$-analog of the Schur-Weyl duality. It had applications to (new) symmetric functions, as well as to the theory of polynomial identities.

Sets of integer partitions correspond to sets of Young tableaux, and these give rise to the corresponding combinatorial sums - with their corresponding asymptotics. Also, the asymptotics of the corresponding combinatorial sum imply interesting relations with the celebrated Mehta-Selberg integral. Surprisingly, that fundamental integral allows the exact computation of the asymptotics of the polynomial identities of matrices.

Mansour: What would guide you in your research? A general theoretical question or a specific problem?

Regev: Usually, it would be a specific problem.

Mansour: What three results related to your work do you consider the most influential in combinatorics during the last thirty years?

Regev: Two of my own research(see footnote 1) and one of Zeilberger: (1) $A \otimes B$ theorem introducing "growth". (2) The Mehta-Selberg integral and the fact that it allows us computing the asymptotics of the codimension sequence $c_{n}(A)$, where $A$ is the algebra of $k \times k$ matrices. But I should probably include the $\mathbb{Z}_{2}$ Schur-Weyl duality (with Berele) with the corresponding hook Schur functions. We sometimes can determine if the corresponding generating is algebraic or not. For instance, mine result with Beckner states that when $k$ is odd number then the generating function is not algebraic. (3) Zeilberger's works with Shalosh B. Ekhad.

Mansour: What are the top three open questions in your list?

Regev: (1) Determine the exact asymptotics $c_{n}\left(M_{k}(F)\right)$ (see footnote ${ }^{1}$ ). Very recently, in a joint work with Berele, we showed a fairly tight sandwich theorem for those invariants. With the notations $c_{n}\left(M_{k}(F)\right) \sim a n^{b} r^{n}$ it follows that $b$ is half-integer, $r$ is a positive integer according to a result of Giambruno- Zaicev, however, there is still no information about the constant $a$. Thus, it is interesting to determine the constants $a$ and $b$. (2) In a joint work with Beckner, we proved that $c_{n}\left(M_{k}(F)\right)$ is non-algebraic if $k$ is odd. My previously mentioned result with Beckner solves partially a problem of R. Stanley. The corresponding question when $k$ is even - is open. The obvious conjecture is: this is true for all $k$; no doubt it is true. (3) Similar works for $c_{n}\left(M_{k}(G)\right.$ ) (see footnote ${ }^{1}$ ) where $G$ is the Grassmann algebra. Also, to determine $a, b$ and $r$ for the verbally prime algebras.

Mansour: What kind of mathematics would you like to see in the next ten-to-twenty years as the continuation of your work?

Regev: I would prefer a more concrete and less so-called "abstract nonsense". Also, I would like to see more results of the collaboration of Zeilberger and Shalosh B. Ekhad ${ }^{3}$.

Mansour: What would you say about some of the major directions in combinatorics for the next two decades?

Regev: The combinatorics of superalgebras ${ }^{4}$. Mansour: Do you think that there are core or mainstream areas in mathematics? Are some topics more important than others?

Regev: No doubt certain areas of mathematics have more prestige than others. Number theory is more appealing than most other areas.

Mansour: What do you think about the dis-

\footnotetext{
${ }^{3}$ See, https://sites.math.rutgers.edu/ zeilberg/pj.html

${ }^{4}$ V.G. Kac, C. Martinez and E. Zelmanov, Graded simple Jordan superalgebras of growth one, Memoirs of the AMS Series 711 , 2001.
} 
tinction between pure and applied mathematics that some people focus on? Is it meaningful at all in your own case? How do you see the relationship between so-called "pure" and "applied" mathematics?

Regev: Such a distinction probably exists, but the intersection of pure and applied mathematics is large. For example, the character theory of $S_{n}$ can later be considered pure, but it is applied in physics.

Mansour: What advice would you give to young people thinking about pursuing a research career in mathematics?

Regev: Try to find a topic with interesting and/or natural applications. It seems that people are getting tired from too abstract and irrelevant applications. Nevertheless, work hard and do not give up.

Mansour: Would you tell us about your interests besides mathematics?

Regev: Playing Baroque music on the Oboe, with a small group of Baroque players. But right now I am not healthy enough for that.

Mansour: Before we close this interview with one of the foremost experts in combinatorics, we would like to ask some more specific mathematical questions. The applications of the representation theory of the symmetric group to the study of algebras satisfying polynomial identities played an important role in your research. You used Young diagrams many times and obtained a series of important results in this field. Would you describe the main ideas and the combinatorial methods you used in this direction?

Regev: Given $n$ and a PI algebra A, we measure how large is the part of non-identities relative to the whole space. A significant simplification is obtained when restricting to multilinear identities. This brings in the representations of $S_{n}$. This yields the sequence of codimensions of $A$. Similarly for the corresponding sequence representation of $S_{n}$. These two sequences determine a lot about the PI algebra $A$, hence the relations to Young diagrams.

Mansour: The study of permutation patterns has seen great advances in the last thirty years. One of the earliest non-trivial result was obtained by you that gives the growth rate of the number of the permutations avoiding a monotone pattern of length $k$. But we still do not know how to enumerate the 1324-avoiding per- mutations. What do you think about the research in this direction?

Regev: In my work, I somehow avoided counting Pattern-Avoiding permutations ["The Stanley-Wilf" Conjecture]. A few years ago I asked Wilf why they made that conjecture? He said: "Because of your results".

Mansour: Would you tell us about your thought process for the proof of one of your most important results? How did you become interested in that problem? How long did it take you to figure out a proof? Did you have a "eureka moment"?

Regev: Eureka moment occurred for the $A \otimes B$ problem (see footnote ${ }^{1,3}$ ) when I realized that even the $G \otimes G$ case is still open.

Mansour: Is there a specific problem you have been working on for many years? What progress have you made?

Regev: Yes, several such problems. For example, (1) with Alan Berele, the asymptotics of the cocharacters of the ring of matrices over a field (see footnote ${ }^{1}$ ). At the end, this was determined by an application of the MehtaSelberg integral. (2) Various statistics on $S_{n}$ were introduced by MacMahon who studied their equidistributions, where with Roichman we extended these theorems to corresponding $q$-analogues. (3) The existence of central polynomials has important consequences for the structure of the algebra. Kaplansky asked if central polynomials on matrices exist. Then Formanek and, indepenedently, Razmislov proved: Yes, they exist. For $k \times k$ matrices, I constructed such central polynomials as follows: split $k^{2} x$ s into $k$ blocks of $x \mathrm{~s}$ of lengths $1,3, \ldots$ and similarly split $k^{2} y$ s. Now shuffle these blocks, and finally alternate the $x \mathrm{~s}$ and independently the $y \mathrm{~s}$. The result is a polynomial $P_{k}\left[x_{1}, \ldots, x_{k^{2}}, y_{1}, \ldots, y_{k^{2}}\right]$ which I introduced nearly 40 years ago. It is easy to see that $P_{k}[x, y]$ is central (i.e. takes central values on $k \times k$ matrices). I checked that these polynomials are non-zero for $k=2$ and $k=3$. Then I conjectured that $P_{k}[x, y]$ is non-zero for all $k$. At the time it seemed an extremely difficult problem. Then Formanek found an incredibly ingenious proof which proved that conjecture. These central polynomials $P_{k}[x, y]$ already found several applications. (Some people call $P_{k}[x, y]$ "the Formanek-Regev" central polynomials in PI Theory.) 
Mansour: Professor Amitai Regev, I would terview on behalf of the journal Enumerative like to thank you for this very interesting in- Combinatorics and Applications. 